INPLASY

PROTOCOL

To cite: Yan et al. The efficacy and neural correlates of ERPbased therapy for OCD \& TS:

A systematic review and metaanalysis. Inplasy protocol

2021120112. doi:

10.37766/inplasy2021.12.0112

Received: 24 December 2021

Published: 24 December 2021

Corresponding author:

Ying Li

liying@bch.com.cn

Author Affiliation:

Department of Psychiatry,

Beijing Children's Hospital,

Capital Medical University,

National Center for Children

Healthy.

Support: No. XTYB201802.

Review Stage at time of this submission: Formal screening of search results against eligibility criteria.

Conflicts of interest:

None declared.

\section{The efficacy and neural correlates of ERP-based therapy for OCD \& TS: A systematic review and meta-analysis}

Yan, J1; Cui, L2; Wen, F3; Yu, L4; Wang, F5; Liu, J6; Wang M7; Cui Y8; Li, Y9.

Review question / Objective: Exposure and response prevention (ERP) is a form of cognitive behavioral therapy (CBT) that can effectively relieve obsessive-compulsive symptoms and tic symptoms in patients with obsessivecompulsive disorder (OCD) and Tourette syndrome (TS). However, the effect size of ERP based therapy is still unclear. Therefore, we will perform a meta-analysis to identify the efficacy of ERP based therapy between OCD patients and control group, TS patients and control group.

Information sources: We will search, with no time restrictions, the following databases for relevant English language literature: PubMed (MEDLINE) and Web of Science.The search terms are as follows: "obsessive-compulsive disorder" or "OCD" or obsessive/compulsive" or "Tourette's syndrome or tics or tic disorders" and "cognitive behavior therapy" or "exposure and response" or "exposure and ritual prevention," or "ERP" or "EX/RP" or "psychotherapy".

INPLASY registration number: This protocol was registered with the International Platform of Registered Systematic Review and Meta-Analysis Protocols (INPLASY) on 24 December 2021 and was last updated on 24 December 2021 (registration number INPLASY2021120112).

\section{INTRODUCTION}

Review question / Objective: Exposure and response prevention (ERP) is a form of cognitive behavioral therapy (CBT) that can effectively relieve obsessive-compulsive symptoms and tic symptoms in patients with obsessive-compulsive disorder (OCD) and Tourette syndrome (TS). However, the effect size of ERP based therapy is still unclear. Therefore, we will perform a metaanalysis to identify the efficacy of ERP 
based therapy between OCD patients and control group, TS patients and control group.

Condition being studied: Obsessivecompulsive disorder (OCD) is a chronic psychiatric disorder characterized by distressing and time-consuming obsessions and compulsions. Obsessions are defined as intrusive and unwanted thoughts, urges, or images, and they are followed by compulsions, which aim to relieve these "uncomfortable feelings". It is reported that the lifetime prevalence of OCD is about $1 \%-3 \%$. Patients are often affected by obsessions and compulsions that interfere in social, at home, educational attainment, and occupational aspects. Exposure and response prevention (ERP) is based on cognitive behavioral therapy (CBT) and is the primary psychological treatment for OCD in children, adolescents, and adults. ERP involves exposure to feared obsessional stimuli while refraining from engaging in compulsive behaviors . Research indicates that about $60 \%-85 \%$ of patients who complete ERP treatment see significant success in alleviating obsessivecompulsive symptoms. Moreover, CBT (including ERP) is recommended as the first-line treatment for mild-to-moderate OCD in youth.Furthermore, ERP is applied not only in patients with OCD but also in patients with Tourette syndrome (TS). TS is characterized by sudden motor movements and/or vocalizations (referred to as tics) for at least 12 months.It has been reported that the worldwide prevalence of TS is nearly $1 \%$. ERP has also been recommended as first-line behavioral therapy in American, Canadian and European guidelines for tic disorders. However, the efficacy of ERP for TS is needed to be clarified, as well as the effect size of ERP based therapy is still unclear.

\section{METHODS}

Participant or population: Patients with OCD or TS/chronic tic disorder will be included.
Intervention: Exposure and response prevention (ERP) is based on cognitive behavioral therapy (CBT) and is the primary psychological treatment for OCD and TS in children, adolescents, and adults.

Comparator: Using other therapies for OCD or TS patients.

Study designs to be included: Randomized clinical trials will be included irrespective of blinding, publication status or language.

Eligibility criteria: Not randomized clinical trails.

Information sources: We will search, with no time restrictions, the following databases for relevant English language literature: PubMed (MEDLINE) and Web of Science.The search terms are as follows: "obsessive-compulsive disorder" or "OCD" or obsessive/compulsive" or "Tourette's syndrome or tics or tic disorders" and "cognitive behavior therapy" or "exposure and response" or "exposure and ritual prevention," or "ERP" or "EX/RP" or "psychotherapy".

Main outcome(s): Yale-Brown ObsessiveCompulsive Symptom Scale (Y-BOCS); Children's Yale-Brown ObsessiveCompulsive Scale (CY-BOCS).

Quality assessment / Risk of bias analysis: we will use Egger's funnel plot to deal with publication bias.

Strategy of data synthesis: We will use randomized effects model to examine the standard mean difference (SMD) of ERP based therapy. If the SMD will between 0.2 and 0.5, it means the efficacy of ERP based therapy is mild-to-moderate, whereas SMD values between 0.5 and 0.8 indicates that the efficacy of ERP based therapy is moderate-to-large. The 12 and forest plots will be used to identify the heterogeneity of ERP. If I 2 is greater than $50 \%$, we will use a random-effects model. We then will use subgroup and meta-regression analyses to explore heterogeneities in the effect size for ERP based therapy. We will consider a p-value $<0.05$ to be statistically significant. 
Subgroup analysis: A subgroup analysis of the pooled SMD of ERP-based therapy for OCD will be conducted to identify the potential source of heterogeneity by different comparisons of ERP based therapy and different age groups.

Sensitivity analysis: We will use sensitivity analysis to explore the heterogeneity of the pooled SMD of ERP based therapy for OCD. This method omits one study at a time and tracks the change in 12 to identify the contribution of each study to the heterogeneity.

Country(ies) involved: China.

Keywords: Exposure and response prevention, Cognitive behavioral therapy, Obsessive-compulsive disorder, Tourette syndrome, tic disorders, meta-analysis.

Contributions of each author:

Author 1 - Junjuan Yan.

Author 2 - Linyu Cui.

Author 3 - Fang Wen.

Author 4 - Liping Yu.

Author 5 - Fang Wang.

Author 6 - Jingran Liu.

Author 7 - Mengyu Wang.

Author 8 - Yonghua Cui.

Author 9 - Ying Li. 